OPEN ACCESS

Edited by:

Hui Yan,

South China Normal University, China

Reviewed by:

Keyu Xia,

Nanjing University, China Jianming Wen,

Kennesaw State University,

United States

*Correspondence:

Jing Wang

pwl1207wj@163.com

Jin-Hui Wu

jhwu@nenu.edu.cn

Specialty section:

This article was submitted to

Quantum Engineering and

Technology,

a section of the journal

Frontiers in Physics

Received: 26 November 2021 Accepted: 20 December 2021

Published: 25 January 2022

Citation:

Jiang B, Yan D, Wang J, Qu D and Wu J-H (2022) Controlled Bistable

Transmission Non-Reciprocity in a

Four-Mode Optomechanical System.

Front. Phys. 9:822694.

doi: 10.3389/fphy.2021.822694

\section{Controlled Bistable Transmission Non-Reciprocity in a Four-Mode Optomechanical System}

\author{
Bo Jiang ${ }^{1}$, Dong Yan ${ }^{2}$, Jing Wang ${ }^{3 *}$, Dezhan $Q u^{4}$ and Jin-Hui Wu ${ }^{1 *}$ \\ ${ }^{1}$ Center for Quantum Sciences and School of Physics, Northeast Normal University, Changchun, China, ${ }^{2}$ School of Science, \\ Changchun University, Changchun, China, ${ }^{3}$ College of Physics, Tonghua Normal University, Tonghua, China, ${ }^{4}$ School of \\ Information Science and Technology, Northeast Normal University, Changchun, China
}

We examine the bistable transmission non-reciprocity in a four-mode optomechanical system, where a mechanical oscillator interacts with one of three coupled optical cavities so as to generate an asymmetric optomechanical non-linearity. Two transmission coefficients in opposite directions are found to exhibit non-reciprocal bistable behaviors due to this asymmetric optomechanical non-linearity as the impedancematching condition is broken for a not too weak input field. Such a bistable transmission non-reciprocity can be well manipulated to exhibit reversible higher isolation ratios in tunable wider ranges of the input field power or one cavity mode detuning by modulating relevant parameters like optical coupling strengths, optomechanical coupling strengths, and mechanical frequencies. This optomechanical system provides a flexible platform for realizing transmission non-reciprocity of weal light signals and may be extended to optical networks with more coupled cavities.

Keywords: transmission non-reciprocity, bistable non-linearity, cavity optomechanics, impedance-matching breaking, optical isolation

\section{INTRODUCTION}

Cavity optomechanics, focusing on enhanced radiation pressure interactions between light fields and mechanical motions, has attracted extensive experimental and theoretical interests owing to its wide applications in processing quantum information, measuring weak signals, and developing new devices [1-9]. Various optomechanical systems have been proposed and fabricated to realize nontrivial tasks and interesting phenomena, such as entanglement generation between cavity and mechanical modes [10-15], ground-state cooling of mechanical resonators [16-20], optomechanically induced transparency (OMIT) and absorption (OMIA) [21-26], Bell nonlocality verification [27], parity-time (PT) symmetry-breaking chaos [28], and tumor structural imaging [29]. We note in particular that optomechanical systems can provide an effective avenue for implementing non-reciprocal devices, like isolators and circulators, required in constructing alloptical communication networks [30-34].

Non-reciprocal devices promise the transmission of signals in one direction while blocking those propagating in the opposite direction and can be utilized to avoid unwanted interference of signals and protect optical sources and systems from noises [35-42]. Breaking reciprocity or time reversal symmetry is typically accomplished with magneto-optical effects [43-45] and has resulted in the emergence of new physics such as topologically protected one-way photonic edge modes [46] and non-reciprocal behaviors in giant atom systems [47, 48]. Unfortunately, magneto-optical effects are not present in standard optoelectronic materials including most metals and semiconductors and may 


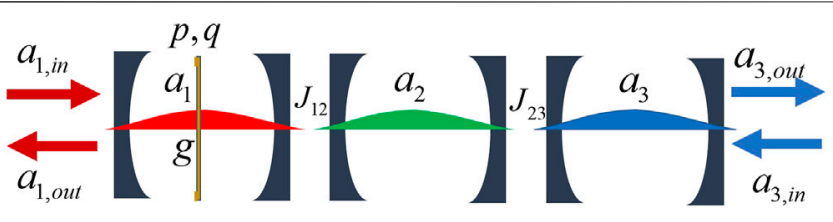

FIGURE 1 | (Color online) Schematic of an optomechanical system consisting of three cavities described by optical modes $a_{1}, a_{2}$, and $a_{3}$ as well as a membrane described by position $q$ and momentum $p$. This system could be driven by an input field $a_{1, \text { in }}$ and exhibit an output field $a_{3, \text { out }}$, or driven by an input field $a_{3, \text { in }}$ and exhibit an output field $a_{1, \text { out }}$. Here, $g$ denotes the optomechanical coupling strength, while $J_{\mathbb{l k}}$ represents the coupling strength between optical modes $a_{l}$ and $a_{k}$.

result in crosstalk and other problems hampering on-chip implementations. This is why non-magnetic approaches for achieving optical non-reciprocity have been extensively studied with significant advances, for example, in chiral atomic systems [49-51] and optomechanical systems [30-34]. The latter includes, for instance, a three-mode optomechanical system with additional gain in one cavity [31] and a two-cavity optomechanical system with a blue-detuned driving [32].

Coupled micro-cavities are essential elements for constructing quantum information networks in that they are scalable via mode swapping or fiber coupling, compatible with mechanical oscillators and other elements, and easy to be controlled by driving fields. With this consideration, here we extend previous works [30-34] to seek more flexible manipulations on optical non-reciprocity by investigating a four-mode optomechanical system with three optical cavities and one mechanical oscillator. This system is found to exhibit an asymmetric optomechanical non-linearity, which then result in staggered bistable behaviors of two opposite-direction transmission coefficients, under the broken impedancematching condition. Numerical results show in particular that quite a few parameters can be modulated on demand to manipulate, in different ways, the upper and lower stable branches of both transmission coefficients. This allows us to tune and widen non-reciprocal ranges in terms of the input power or a cavity detuning on the one hand, while improve isolation ratio and reverse isolation direction with respect to transmission coefficients on the other hand.

\section{MODEL AND EQUATIONS}

We consider a cavity optomechanical system consisting of three optical modes described by annihilation operators $a_{1}, a_{2}$, and $a_{3}$ and a mechanical mode described by position operator $q$ and momentum operator $p$, as shown in Figure 1. These optical and mechanical modes exhibit frequencies $\omega_{1}, \omega_{2}, \omega_{3}$, and $\omega_{m}$, respectively. The 2 nd optical mode is coupled to the $1 s t$ optical mode with strength $J_{12}$, while to the $3 r d$ optical mode with strength $J_{23}$, in a linear way controlled via the in-between waveguide or fiber. The mechanical mode is coupled only to the 1 st optical mode with single-photon optomechanical coupling strength $g$. A driving field of frequency $\omega_{d}$ is applied to excite the 1st optical mode with annihilation operator $a_{1, \text { in }}$ or the $3 r d$ optical mode with annihilation operator $a_{3, i n}$. With these considerations, we can write down the following Hamiltonian $(\hbar=1)$ :

$$
\begin{aligned}
H & =\omega_{1} a_{1}^{\dagger} a_{1}+\omega_{2} a_{2}^{\dagger} a_{2}+\omega_{3} a_{3}^{\dagger} a_{3}+\frac{1}{2} \omega_{m}\left(p^{2}+q^{2}\right) \\
& +g a_{1}^{\dagger} a_{1} q+J_{12}\left(a_{1}^{\dagger} a_{2}+a_{2}^{\dagger} a_{1}\right)+J_{23}\left(a_{2}^{\dagger} a_{3}+a_{3}^{\dagger} a_{2}\right) \\
& +i \sqrt{\gamma_{1, e}}\left(a_{1}^{\dagger} a_{1, i n} e^{-i \omega_{d} t}-a_{1} a_{1, i n}^{\dagger} e^{i \omega_{d} t}\right) \\
& +i \sqrt{\gamma_{3, e}}\left(a_{3}^{\dagger} a_{3, i n} e^{-i \omega_{d} t}-a_{3} a_{3, \text { in }}^{\dagger} e^{i \omega_{d} t}\right),
\end{aligned}
$$

where $\gamma_{j, e}$ has been taken as the coupling constant to the driving field, that is, the external decay rate, of the $j$ th optical mode. It is worth noting that the $j$ th optical mode also exhibits an intrinsic decay rate $\gamma_{j, i}$ so that its total decay rate turns out to be $\gamma_{j}=\gamma_{j, i}+$ $\gamma_{j, e}$. Then we can define $\eta_{j}=\gamma_{j, e} / \gamma_{j}$ as an effective coupling ratio with $\eta_{j}=0$ denoting a vanishing coupling, while $\eta_{j}=1$ denoting the maximal coupling. To be more specific, our optomechanical system may be implemented either with a vibrational membrane coupled to one of three Fabry-Pérot cavities, or with an optomechanical crystal coupled to one of three photonic crystal cavities [52].

In the rotating frame of the driving frequency $\omega_{d}$, it is viable to attain from the Hamiltonian in Eq. 1 the following quantum Langevin equations (QLEs):

$$
\begin{aligned}
\partial_{t} a_{1}= & -\left(\gamma_{1} / 2+i \Delta_{1}\right) a_{1}-i g q a_{1}-i J_{12} a_{2} \\
& +\sqrt{\gamma_{1, e}} a_{1, i n}+\sqrt{\gamma_{1, i}} a_{1, v a c}, \\
\partial_{t} a_{2}= & -\left(\gamma_{2} / 2+i \Delta_{2}\right) a_{2}-i J_{12} a_{1}-i J_{23} a_{3} \\
& +\sqrt{\gamma_{2, i}} a_{2, v a c} \\
\partial_{t} a_{3}= & -\left(\gamma_{3} / 2+i \Delta_{3}\right) a_{3}-i J_{23} a_{2} \\
& +\sqrt{\gamma_{3, e}} a_{3, i n}+\sqrt{\gamma_{3, i}} a_{3, v a c}, \\
\partial_{t} q= & \omega_{m} p \\
\partial_{t} p= & -\omega_{m} q-g a_{1}^{\dagger} a_{1}-\gamma_{m} p+\xi,
\end{aligned}
$$

where $\Delta_{j}=\omega_{j}-\omega_{d}$ is defined as the detuning of the $j$ th optical mode to the driving field, while $\gamma_{m}$ refers to the decay rate of the mechanical mode. In addition, we have used $a_{1, v a c}, a_{2, v a c}, a_{3, v a c}$, and $\xi$ to denote the input quantum noise operators with zero mean values $\left\langle a_{1, v a c}\right\rangle=0,\left\langle a_{2, v a c}\right\rangle=0,\left\langle a_{3, v a c}\right\rangle=0$, and $\langle\xi\rangle$ $=0$ [54].

Each operator of the optical and mechanical modes can be split into a classical mean value and a quantum fluctuation as usual. That means, we can set $a_{j}=\alpha_{j}+\delta a_{j}, a_{j, \text { in }}=\alpha_{j, \text { in }}+\delta a_{j, \text { in }}$, $q=\bar{q}+\delta q$, and $p=\bar{p}+\delta p$ with the ansatz $\alpha_{j}=\left\langle a_{j}\right\rangle, \alpha_{j, \text { in }}=$ $\left\langle a_{j, i n}\right\rangle, \bar{q}=\langle q\rangle$, and $\bar{p}=\langle p\rangle$. In the limit of a much stronger optical driving than the optomechanical coupling, that is, $\sqrt{\gamma_{j, e}}\left|\alpha_{j, i n}\right| \gg \gamma_{j} \gg g$, the classical mean values and the fluctuation operators can be treated separately. Then we can determine the classical mean values, in the steady state $\left(\partial_{t} \alpha_{i}=\partial_{t} \bar{p}=\partial_{t} \bar{q}=0\right)$, via the following equations:

$$
\begin{aligned}
& 0=-\left(\gamma_{1} / 2+i \Delta_{1}\right) \alpha_{1}-i g \bar{q} \alpha_{1}-i J_{12} \alpha_{2}+\sqrt{\gamma_{1, e}} \alpha_{1, i n}, \\
& 0=-\left(\gamma_{2} / 2+i \Delta_{2}\right) \alpha_{2}-i J_{12} \alpha_{1}-i J_{23} \alpha_{3}, \\
& 0=-\left(\gamma_{3} / 2+i \Delta_{3}\right) \alpha_{3}-i J_{23} \alpha_{2}+\sqrt{\gamma_{3, e}} \alpha_{3, i n}, \\
& 0=\bar{p}, \\
& 0=-\omega_{m} \bar{q}-g\left|\alpha_{1}\right|^{2},
\end{aligned}
$$

where the mean field approximation $\left\langle q \alpha_{1}\right\rangle \approx\langle q\rangle\left\langle\alpha_{1}\right\rangle$ has been taken into account. It is not difficult to see that the first $\left(\alpha_{1}\right)$ and 
third $\left(\alpha_{3}\right)$ optical modes are not reciprocal because the mean position $\bar{q}$ of the mechanical mode is just coupled to the mean amplitude $\alpha_{1}$ of the first optical mode via $-i g \bar{q} \alpha_{1}$ with $\bar{q}=-g\left|\alpha_{1}\right|^{2} / \omega_{m}$. That means, the aforementioned equations do not remain unchanged if we exchange subscripts " 1 " and " 3 ." Therefore, a transmission non-reciprocity is expected to occur no matter the driving field comes from either one direction $\left(\alpha_{1, \text { in }} \neq 0\right.$ or $\left.\alpha_{3, \text { in }} \neq 0\right)$ or both directions $\left(\alpha_{1, \text { in }} \neq 0\right.$ and $\left.\alpha_{3, \text { in }} \neq 0\right)$. With these classical mean values in hand, we can attain a set of linearized QLEs for the fluctuation operators in the matrix form

$$
\frac{\partial f}{\partial t}=A f+\zeta
$$

by introducing two column vectors

$$
\begin{aligned}
& f=\left(\delta a_{1}, \delta a_{1}^{\dagger}, \delta a_{2}, \delta a_{2}^{\dagger}, \delta a_{3}, \delta a_{3}^{\dagger}, \delta q, \delta p\right)^{T}, \\
& \zeta=\left(\delta A_{1, \text { in }}, \delta A_{1, \text { in }}^{\dagger}, \delta A_{2, \text { in }}, \delta A_{2, i n}^{\dagger}, \delta A_{3, \text { in }}, \delta A_{3, \text { in }}^{\dagger}, 0, \xi\right)^{T}
\end{aligned}
$$

and a coefficient matrix

$$
A=\left[\begin{array}{cccccccc}
-\left(\frac{\gamma_{1}}{2}+i \Delta_{1}^{\prime}\right) & 0 & -i J_{12} & 0 & 0 & 0 & -i g \alpha_{1} & 0 \\
0 & -\left(\frac{\gamma_{1}}{2}-i \Delta_{1}^{\prime}\right) & 0 & i J_{12} & 0 & 0 & i g \alpha_{1}^{*} & 0 \\
-i J_{12} & 0 & -\left(\frac{\gamma_{2}}{2}+i \Delta_{2}\right) & 0 & -i J_{23} & 0 & 0 & 0 \\
0 & i J_{12} & 0 & -\left(\frac{\gamma_{2}}{2}-i \Delta_{2}\right) & 0 & i J_{23} & 0 & 0 \\
0 & 0 & -i J_{23} & 0 & -\left(\frac{\gamma_{3}}{2}+i \Delta_{3}\right) & 0 & 0 & 0 \\
0 & 0 & 0 & i J_{23} & 0 & -\left(\frac{\gamma_{3}}{2}-i \Delta_{3}\right) & 0 & 0 \\
0 & 0 & 0 & 0 & 0 & 0 & 0 & \omega_{m} \\
-g \alpha_{1}^{*} & -g \alpha_{1} & 0 & 0 & 0 & 0 & -\omega_{m} & -\gamma_{m}
\end{array}\right] \text {, }
$$

where we have further defined $\delta A_{1, \text { in }}=\sqrt{\gamma_{1, e}} \delta a_{1, \text { in }}+\sqrt{\gamma_{1, i}} a_{1, v a c}$, $\delta A_{2, \text { in }}=\sqrt{\gamma_{2, i}} a_{2, \text { vac }}, \quad \delta A_{3, \text { in }}=\sqrt{\gamma_{3, e}} \delta a_{3, \text { in }}+\sqrt{\gamma_{3, i}} a_{3, \text { vac }}$, and $\Delta_{1}^{\prime}=\Delta_{1}+g \bar{q}$. Our optomechanical system can work in the stable regime only if all the eigenvalues of matrix $A$ are negative in their real parts. This problem is difficult or impossible to be solved analytically but can be by numerically examined via the Routh-Hurwitz criterion [53] as adopted later.

In the following, we consider two specific cases where the driving field of amplitude $s_{i n}=\sqrt{p_{i n} /\left(\hbar \omega_{d}\right)}$ and power $p_{\text {in }}$ is input just from the 1st optical mode with $\alpha_{1, \text { in }}=s_{\text {in }}$ and $\alpha_{3, \text { in }}=0$ (I), or just from the $3 r d$ optical mode with $\alpha_{1, \text { in }}=0$ and $\alpha_{3, \text { in }}=s_{\text {in }}$ (II). In case $(I)$, it is easy to attain from Eq. 3 that

$$
\begin{aligned}
& \alpha_{3}=\frac{-J_{12} J_{23} \alpha_{1}}{\left(\gamma_{2} / 2+i \Delta_{2}\right)\left(\gamma_{3} / 2+i \Delta_{3}\right)+J_{23}^{2}}, \\
& \alpha_{1}=\frac{-J_{12} J_{23} \alpha_{3}+\left(\gamma_{2} / 2+i \Delta_{2}\right) \sqrt{\gamma_{1, e}} s_{i n}}{\left(\gamma_{2} / 2+i \Delta_{2}\right)\left(\gamma_{1} / 2+i \Delta_{1}-i U\left|\alpha_{1}\right|^{2}\right)+J_{12}^{2}},
\end{aligned}
$$

with $U=g^{2} / \omega_{m}$ characterizing the non-linear optomechanical interaction. Considering the input-output relation $\alpha_{3, \text { out }}=$ $\sqrt{\gamma_{3, e}} \alpha_{3}[55,56]$, we finally attain

$$
(\Gamma / 2+i \bar{\Delta}) \alpha_{3, \text { out }}+i U_{31}\left|\alpha_{3, \text { out }}\right|^{2} \alpha_{3, \text { out }}=\varepsilon_{\text {eff }}
$$

In this equation, we have introduced the effective damping rate $\Gamma$, detuning $\bar{\Delta}$, non-linear interaction strength $U_{31}$, and driving amplitude $\varepsilon_{\text {eff }}$ by setting

$$
\begin{aligned}
\Gamma= & \frac{A\left(\gamma_{2} \gamma_{3} / 4-\Delta_{2} \Delta_{3}+J_{23}^{2}\right)+B\left(\gamma_{2} \Delta_{3}+\gamma_{3} \Delta_{2}\right)}{\left|\left(\gamma_{2} / 2+i \Delta_{2}\right)\left(\gamma_{3} / 2+i \Delta_{3}\right)+J_{23}^{2}\right|^{2}}, \\
\bar{\Delta} & =\frac{B\left(\gamma_{2} \gamma_{3} / 4-\Delta_{2} \Delta_{3}+J_{23}^{2}\right)-A\left(\gamma_{2} \Delta_{3}+\gamma_{3} \Delta_{2}\right) / 4}{\left|\left(\gamma_{2} / 2+i \Delta_{2}\right)\left(\gamma_{3} / 2+i \Delta_{3}\right)+J_{23}^{2}\right|^{2}}, \\
\varepsilon_{e f f} & =\frac{-J_{12} J_{23} \sqrt{\gamma_{1, e} \gamma_{3, e}} s_{i n}}{\left(\gamma_{2} / 2+i \Delta_{2}\right)\left(\gamma_{3} / 2+i \Delta_{3}\right)+J_{23}^{2}}, \\
U_{31} & =\frac{-U\left|\left(\gamma_{2} / 2+i \Delta_{2}\right)\left(\gamma_{3} / 2+i \Delta_{3}\right)+J_{23}^{2}\right|^{2}}{J_{12}^{2} J_{23}^{2} \gamma_{3, e}},
\end{aligned}
$$

with newly defined coefficients

$$
\begin{aligned}
A & =\gamma_{3}\left(\gamma_{1} \gamma_{2} / 4-\Delta_{1} \Delta_{2}\right)-\Delta_{3}\left(\Delta_{1} \gamma_{2}+\Delta_{2} \gamma_{1}\right) \\
& +J_{23}^{2} \gamma_{1}+J_{12}^{2} \gamma_{3}, \\
B & =\gamma_{3}\left(\Delta_{1} \gamma_{2}+\Delta_{2} \gamma_{1}\right) / 4+\Delta_{3}\left(\gamma_{1} \gamma_{2} / 4-\Delta_{1} \Delta_{2}\right) \\
& +J_{23}^{2} \Delta_{1}+J_{12}^{2} \Delta_{3} .
\end{aligned}
$$

In case $(I I)$, we attain via a similar procedure.

$$
\begin{aligned}
\alpha_{1}= & \frac{-J_{12} J_{23} \alpha_{3}}{\left(\gamma_{1} / 2+i \Delta_{1}-i U\left|\alpha_{1}\right|^{2}\right)\left(\gamma_{2} / 2+i \Delta_{2}\right)+J_{12}^{2}}, \\
\alpha_{3}= & \frac{-J_{12} J_{23} \alpha_{1}+\left(\gamma_{2} / 2+i \Delta_{2}\right) \sqrt{\gamma_{3, e}} s_{i n}}{\left(\gamma_{2} / 2+i \Delta_{2}\right)\left(\gamma_{3} / 2+i \Delta_{3}\right)+J_{23}^{2}},
\end{aligned}
$$

which, when substituting into the input-output relation $\alpha_{1, \text { out }}=\sqrt{\gamma_{1, e}} \alpha_{1}$, finally yields

$$
(\Gamma / 2+i \bar{\Delta}) \alpha_{1, \text { out }}+i U_{13}\left|\alpha_{1, \text { out }}\right|^{2} \alpha_{1, \text { out }}=\varepsilon_{e f f},
$$

with a new effective non-linear interaction strength $U_{13}=-U /$ $\gamma_{1, e}$, clearly different from $U_{31}$.

For convenience, we now translate Eqs 8, 12 into a unified form in terms of $X_{i}=\left|\alpha_{i, \text { out }}\right|^{2}$

$$
\left(\Gamma^{2} / 4+\bar{\Delta}^{2}\right) X_{i}+2 \bar{\Delta} U_{e f f} X_{i}^{2}+U_{e f f}^{2} X_{i}^{3}=\left|\varepsilon_{e f f}\right|^{2},
$$

with $U_{\text {eff }}=U_{13}$ for $X_{1}=\left|\alpha_{1, \text { out }}\right|^{2}$, while $U_{\text {eff }}=U_{31}$ for $X_{3}=\left|\alpha_{3, \text { out }}\right|^{2}$. This non-linear equation indicates that $X_{i}$ can take three real values, corresponding to the bistability of output against input, under appropriate conditions. One way for determining the bistable region is to take a derivative of Eq. 13 with respect to $X_{i}$, yielding

$$
\left(\Gamma^{2} / 4+\bar{\Delta}^{2}\right)+4 \bar{\Delta} U_{e f f} X_{i}+3 U_{e f f}^{2} X_{i}^{2}=0,
$$

whose two positive roots

$$
X_{i}^{ \pm}=\frac{-4 \bar{\Delta} \mp \sqrt{4 \bar{\Delta}^{2}-3 \Gamma^{2}}}{6 U_{e f f}}>0,
$$

restricted by $\bar{\Delta}<-\sqrt{3} \Gamma / 2$ referring to two turning points of the bistable region. That means, the solution of Eq. 15 takes three branches in the bistable region of $X_{i}^{-} \leq X_{i} \leq X_{i}^{+}$. The intermediate branch is known to be definitely unstable because it corresponds to the maximum (not minimum) point in an effective potential, while the upper and lower branches are usually stable, for example, in a non-linear Kerr medium [57, 58]. In our optomechanical system, the upper branch may also be unstable as the mechanical mode exhibits a negative effective 
damping rate owing to a heating effect in the blue-detuned or strong-driving regime, which will be numerically examined via the Routh-Hurwitz criterion [53].

The expected non-linear bistability is straightforward to be examined by two transmission coefficients:

$$
\begin{aligned}
& T_{31}=\left|\alpha_{3, \text { out }} / \alpha_{1, \text { in }}\right|^{2}, \\
& T_{13}=\left|\alpha_{1, \text { out }} / \alpha_{3, \text { in }}\right|^{2},
\end{aligned}
$$

referring, respectively, to a transport from the 1st optical mode to the $3 r d$ optical mode and that from the $3 r d$ optical mode to the $1 s t$ optical mode. Considering that $U_{31}$ and $U_{13}$ have different expressions, we know from Eqs 8, 12 that $a_{3, \text { out }} \neq a_{1, \text { out }}$ in general and therefore $T_{31} \neq T_{13}$ for light signals of amplitudes $a_{1, \text { in }}=a_{2, \text { in }}=s_{\text {in }}$ input from the opposite sides of our optomechanical system. The efficiency of such a nonreciprocal transport can be quantified by defining

$$
I_{t r}=10 \log \left(T_{31} / T_{13}\right),
$$

as the isolation ratio. We should note however that it is also possible to have $U_{31}=U_{13}$ in the case of

$$
\gamma_{1, e}=\frac{J_{12}^{2} J_{23}^{2} \gamma_{3, e}}{\left|\left(\gamma_{2} / 2+i \Delta_{2}\right)\left(\gamma_{3} / 2+i \Delta_{3}\right)+J_{23}^{2}\right|^{2}},
$$

referred to as the impedance-matching condition, from which it is viable to get a critical coupling strength

$$
J_{23}^{c}=\sqrt{\frac{\gamma_{3, e} J_{12}^{2}-2 \gamma_{1, e}\left(\gamma_{2} \gamma_{3} / 4-\Delta_{2} \Delta_{3}\right) \pm \sqrt{C}}{2 \gamma_{1, e}}},
$$

with $C=\gamma_{3, e}^{2} J_{12}^{4}-\gamma_{1, e} \gamma_{3, e} J_{12}^{2}\left(\gamma_{2} \gamma_{3}-4 \Delta_{2} \Delta_{3}\right)-\gamma_{1, e}^{2}\left(\gamma_{2} \Delta_{3}-\Delta_{2} \gamma_{3}\right)$ independent of input power $p_{\text {in }}$. It is clear that in the case of $J_{23} \neq J_{23}^{c}$, the impedance-matching condition will be broken, and we could have unequal (optomechanical) non-linear interaction strengths $U_{31} \neq U_{13}$. This would result in the optical transmission non-reciprocity characterized by $T_{31} \neq T_{13}$ and thus $I_{\text {tran }} \neq 0$.

\section{RESULTS AND DISCUSSION}

In this section, we examine the effects of relevant tunable parameters on the non-reciprocal bistable transmission of light signals input from the opposite sides of our optomechanical system via numerical calculations. Most parameters are chosen based on two recent works and accessible in up-to-date experiments [31, 44], among which $\gamma_{1} / 2 \pi=1.0 \mathrm{GHz}, \gamma_{2} / 2 \pi=$ $0.5 \mathrm{GHz}, \gamma_{3} / 2 \pi=4.5 \mathrm{GHz}, \eta_{1}=\eta_{2}=\eta_{3}=0.9, \omega_{d} / 2 \pi=300 \mathrm{THz}$, and $\gamma_{m} / 2 \pi=6.0 \mathrm{MHz}$ are fixed in the following discussions. Numerical results will be shown in two cases where transmission coefficients $T_{31}$ and $T_{13}$ are plotted against input power $p_{\text {in }}$ and detuning $\Delta_{1}$, respectively, as they are much easier to modulate in regard of real applications. The main difficulty relevant to an experimental realization of our proposal lies in that the accurate preparation and arrangement of three (micro)coupled cavities of identical optical modes while different decay rates. A (micro) mechanical oscillator of proper resonant frequency and
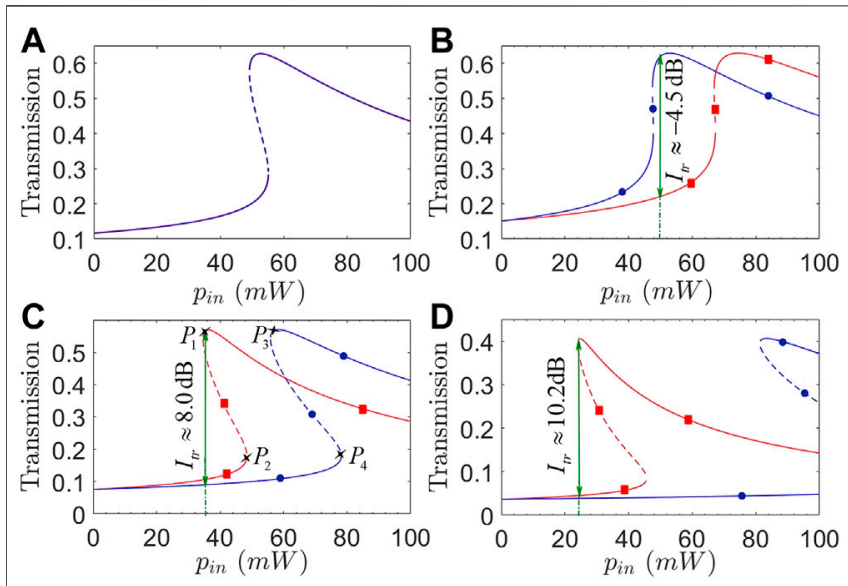

FIGURE 2 | (Color online) Transmission coefficients $T_{31}$ (red squares) and $T_{13}$ (blue circles) against input power $p_{\text {in }}$ with (A) $J_{23} / 2 \pi=1.725 \mathrm{GHz}$; (B) $J_{23} / 2 \pi=2.0 \mathrm{GHz}$; (C) $J_{23} / 2 \pi=1.4 \mathrm{GHz}$; (D) $J_{23} / 2 \pi=1.0 \mathrm{GHz}$. Solid and dashed parts of each curve refer to stable and unstable regions, respectively. Other parameters are $\Delta_{1} / 2 \pi=\Delta_{2} / 2 \pi=4.5 \mathrm{GHz}, \Delta_{3} / 2 \pi=$ $1.5 \mathrm{GHz}, g / 2 \pi=0.9 \mathrm{MHz}, \omega_{m} / 2 \pi=10 \mathrm{GHz}$, and $J_{12} / 2 \pi=3.0 \mathrm{GHz}$, except those at the beginning of Section 3 .

optomechanical coupling strength may also be hard to be integrated with one (micro)optical cavity.

\subsection{Non-Reciprocal Transmission Against Input Power}

In Figure 2, we plot transmission coefficients $T_{31}$ and $T_{13}$ as a function of input power $p_{i n}$ for different optical coupling strengths $J_{23}$. Figure $\mathbf{2 A}$ shows that transmission nonreciprocity (i.e., $T_{13} \neq T_{31}$ or $I_{\text {tran }} \neq 0$ ) cannot be attained as the impedance-matching condition is well satisfied with $J_{23} / 2 \pi=J_{23}^{c} / 2 \pi \simeq 1.725 \mathrm{GHz}$, though our optomechanical system works in the bistable regime. Increasing or decreasing $J_{23}$ to deviate from the critical value $J_{23}^{c}$, we can see from Figures 2B-D that transmission non-reciprocity occurs with different isolation ratios for different input powers. We have, in particular, that $I_{t r} \approx-4.5 \mathrm{~dB}$ for $p_{\text {in }}=50 \mathrm{~mW}$ in the case of $J_{23} / 2 \pi=2.0 \mathrm{GHz}$, $I_{t r} \approx 8.0 \mathrm{~dB}$ for $p_{i n}=36 \mathrm{~mW}$ in the case of $J_{23} / 2 \pi=1.4 \mathrm{GHz}$, and $I_{t r}$ $\approx 10.2 \mathrm{~dB}$ for $p_{\text {in }}=25 \mathrm{~mW}$ in the case of $J_{23} / 2 \pi=1.0 \mathrm{GHz}$. These results indicate that it is viable to reverse the transmission nonreciprocity from $I_{t r}<0$ to $I_{t r}>0\left(I_{t r}>0\right.$ to $\left.I_{t r}<0\right)$ as $J_{23}$ is decreased (increased) to cross the critical value $J_{23}^{c}$, and we can attain higher isolation ratios in wider bistable regions by modulating $J_{23}$ to be more deviating from the critical value $J_{23}^{c}$. Taking Figure $2 \mathrm{C}$ as an example, it is also important to note that we should choose to work in the region between turning points $P_{2}$ and $P_{4}$ as $p_{i n}$ is increased from a small value, while between $P_{1}$ and $P_{3}$ as $p_{\text {in }}$ is decreased from a large value. This promises for attaining a more efficient transmission nonreciprocity corresponding to a larger $\left|I_{t r}\right|$ because it can be evaluated with the upper branch of $T_{31}$ and the lower branch of $T_{13}$. Otherwise, $T_{31}$ and $T_{13}$ will both work in the lower or 

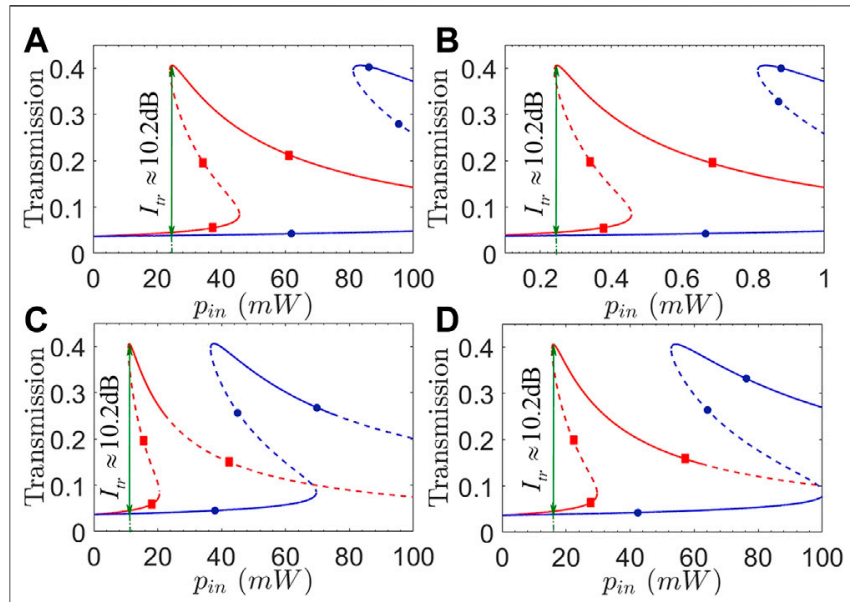

FIGURE 3 | (Color online) Transmission coefficients $T_{31}$ (red squares) and $T_{13}$ (blue circles) against input power $p_{\text {in }}$ with (A) $g / 2 \pi=0.9 \mathrm{MHz}$; (B) $g / 2 \pi$ $=9.0 \mathrm{MHz}$; (C) $\omega_{m} / 2 \pi=4.5 \mathrm{GHz}$; (D) $\omega_{m} / 2 \pi=6.5 \mathrm{GHz}$. Solid and dashed parts of each curve refer to stable and unstable regions, respectively. Other parameters are the same as in Figure 2, except $J_{23} / 2 \pi=1.0 \mathrm{GHz}$.
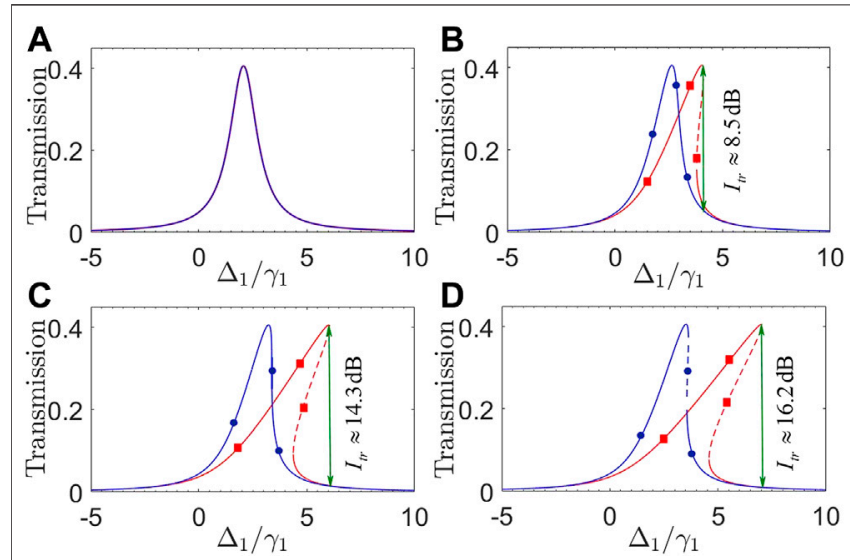

FIGURE 5 | (Color online) Transmission coefficients $T_{31}$ (red squares) and $T_{13}$ (blue circles) against detuning $\Delta_{1}$ with (A) $p_{\text {in }}=0.1 \mathrm{~mW}$; (B) $p_{i n}=$ $20 \mathrm{~mW}$; (C) $p_{\text {in }}=40 \mathrm{~mW}$; (D) $p_{\text {in }}=50 \mathrm{~mW}$. Solid and dashed parts of each curve refer to stable and unstable regions, respectively. Other parameters are $\Delta_{2} / 2 \pi=4.5 \mathrm{GHz}, \Delta_{3} / 2 \pi=1.5 \mathrm{GHz}, g / 2 \pi=0.9 \mathrm{MHz}, \omega_{m} / 2 \pi=$ $10 \mathrm{GHz}, J_{12} / 2 \pi=3.0 \mathrm{GHz}$, and $J_{23}=1.0 \mathrm{GHz}$, except those at the beginning of Section 3.

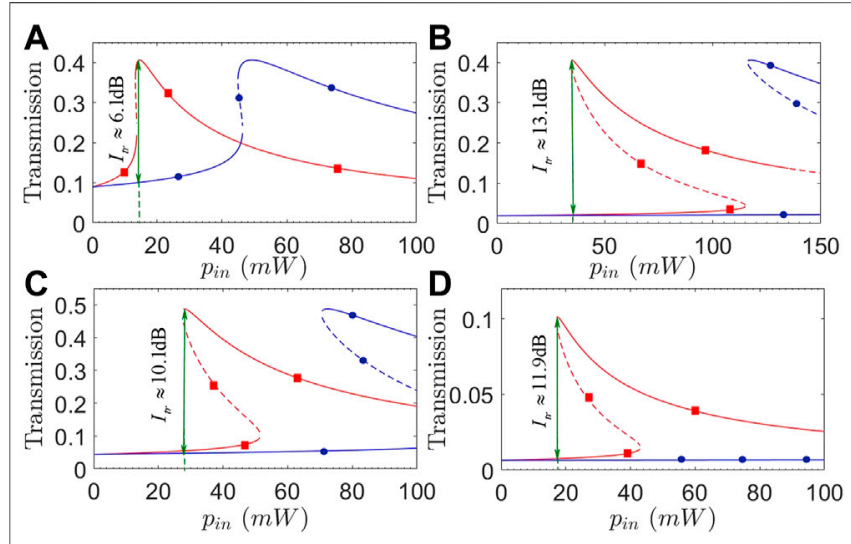

FIGURE 4 | (Color online) Transmission coefficients $T_{31}$ (red squares) and $T_{13}$ (blue circles) against input power $p_{\text {in }}$ with (A) $\Delta_{1} / 2 \pi=3.5 \mathrm{GHz}$; (B) $\Delta_{1} /$ $2 \pi=5.5 \mathrm{GHz}$; (C) $\Delta_{3} / 2 \pi=0.1 \mathrm{GHz}$; (D) $\Delta_{3} / 2 \pi=6.0 \mathrm{GHz}$. Solid and dashed parts of each curve refer to stable and unstable regions, respectively. Other parameters are the same as in Figure 2, except $J_{23} / 2 \pi=1.0 \mathrm{GHz}$.

upper branch to result in well suppressed transmission nonreciprocity of smaller or vanishing $\left|I_{t r}\right|$.

Comparing Eqs 8, 12, it is easy to see that the transmission nonreciprocity will be attained as long as we have $U_{13} \neq U_{31}$, which requires not only a broken impedance-matching condition but also $U=g^{2} / \omega_{m} \neq 0$. Thus, it is essential to examine in Figure 3 different effects of optomechanical coupling strength $g$ and mechanical frequency $\omega_{m}$ on transmission coefficients $T_{31}$ and $T_{13}$ plotted against input power $p_{i n}$. Figures $\mathbf{3 A}, \mathbf{B}$ show that as $g$ is enhanced by one order, $p_{i n}$ required for observing the transmission nonreciprocity (in the bistable region where $T_{13}$ and $T_{31}$ work in the lower and upper branches, respectively) is reduced by two orders without changing the maximal isolation ratio $I_{t r} \approx 10.2 \mathrm{~dB}$. That means the observed transmission non-reciprocity exhibits an inverse dependence on input power $p_{i n}$ and optomechanical coupling strength $g$. Figures 3C,D further show that the non-reciprocal region in terms of $p_{i n}$ is not so sensitive to $\omega_{m}$ though this region can be enlarged in the case of a larger $\omega_{m}$. It is more important to note that the upper branches of $T_{31}$ and $T_{13}$ may not be always stable and a larger $\omega_{m}$ is helpful to reduce the unstable regions. These findings tell us how to choose $g$ and $\omega_{m}$ for attaining a wide enough nonreciprocal region corresponding to low enough input powers.

Considering that the driving field and relevant optical modes are easy to be modulated in frequency, we plot in Figure 4 transmission coefficients $T_{31}$ and $T_{13}$ as a function of input power $p_{\text {in }}$ for different detunings $\Delta_{1}$ and $\Delta_{3}$. We can see from Figures $4 \mathbf{A}, \mathbf{B}$ that the isolation ratio may be evidently improved in a wider non-reciprocal region by choosing a slightly larger $\Delta_{1}$ to well suppress the lower branches of $T_{31}$ and $T_{13}$, while leaving the upper branches unchanged yet in magnitude. To be more specific, we have $I_{t r} \approx 6.1 \mathrm{~dB}$ for $p_{\text {in }}=14 \mathrm{~mW}$ with $\Delta_{1} / 2 \pi=3.5 \mathrm{GHz}$, while $I_{t r} \approx 13.1 \mathrm{~dB}$ for $p_{\text {in }}=35 \mathrm{~mW}$ with $\Delta_{1} / 2 \pi$ $=5.5 \mathrm{GHz}$. Figures 4C,D show instead that a significant increase of $\Delta_{3}$, though can result in a wider non-reciprocal region, will not change the isolation ratio too much as the upper and lower branches are suppressed to the roughly same extent. We also note from Figure 4B that the upper branch of $T_{31}$ starts to become unstable at $p_{\text {in }} \gtrsim 140 \mathrm{~mW}$ for $\Delta_{1}=5.5 \mathrm{GHz}$. It is thus clear that detunings $\Delta_{1}$ and $\Delta_{3}$ play different roles in manipulating the transmission nonreciprocity and can be jointly modulated for observing an ideal transmission non-reciprocity with larger isolation ratios and well suppressed lower branches for moderate input powers.

\subsection{Non-Reciprocal Transmission Against Detuning}

We first plot in Figure 5 transmission coefficients $T_{31}$ and $T_{13}$ as a function of detuning $\Delta_{1}$ for different input powers $p_{i n}$. As the 

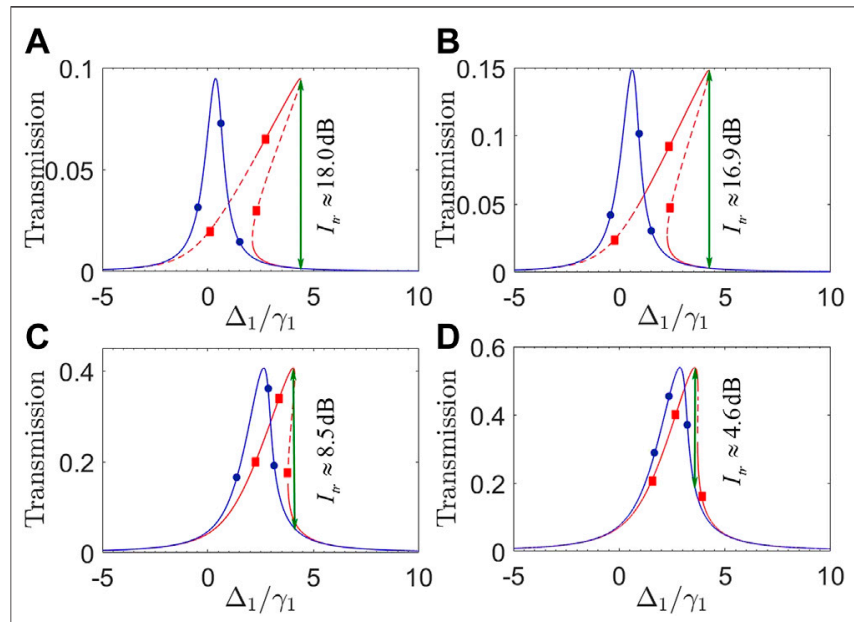

FIGURE 6 | (Color online) Transmission coefficients $T_{31}$ (red squares) and $T_{13}$ (blue circles) against detuning $\Delta_{1}$ with (A) $J_{12} / 2 \pi=1.0 \mathrm{GHz}$; (B) $J_{12} / 2 \pi$ $=1.3 \mathrm{GHz}$; (C) $J_{23} / 2 \pi=1.0 \mathrm{GHz}$; (D) $J_{23} / 2 \pi=1.3 \mathrm{GHz}$. Solid and dashed parts of each curve refer to stable and unstable regions, respectively. Other parameters are the same as in Figure 5, except $p_{\text {in }}=20 \mathrm{~mW}$.

input power is very low (i.e., $p_{\text {in }}=0.1 \mathrm{~mW}$ ), Figure $5 \mathbf{A}$ shows that $T_{31}$ and $T_{13}$ overlap well with a symmetric peak centered at $\Delta_{1} \approx$ $\left(J_{12}+J_{23}\right) / 2=2 \mathrm{GHz}$, therefore leading to a vanishing transmission non-reciprocity. This can be attributed to the fact that both Eqs 8, 12 reduce to $(\Gamma / 2+i \bar{\Delta}) \alpha_{j, \text { out }} \approx \varepsilon_{\text {eff }}$ when $U_{j j^{\prime}}\left|\alpha_{j, \text { out }}\right|^{2}$ with $j+j^{\prime}=4$ is much smaller than $\bar{\Delta}$. As the input power increases to be large enough, we find from Figures 5B-D that $T_{31}$ and $T_{13}$ start to exhibit different bistable behaviors and thus become distinguishable on the side of $\Delta_{1}>\left(J_{12}+J_{23}\right) / 2$ because $U_{13}$ and $U_{31}$ always take negative values. That means the deviation of a transmission peak from its original position may serve as a good estimation on the strength of non-linear optomechanical interaction. To be more specific, the input power $p_{\text {in }}$ has less influence on $T_{13}$ than $T_{31}$ so that the transmission non-reciprocity occurs and becomes more and more evident as $p_{i n}$ increases. It is also noted that a larger input power always results in a wider bistable region with a higher isolation ratio at the right turning point of $T_{31}: I_{t r} \approx 8.5 \mathrm{~dB}$ at $\Delta_{1} / \gamma_{1}=4.0$ for $p_{\text {in }}=20 \mathrm{~mW} ; I_{\text {tr }} \approx 14.3 \mathrm{~dB}$ at $\Delta_{1} / \gamma_{1}=6.0$ for $p_{\text {in }}=$ $40 \mathrm{~mW} ; I_{t r} \approx 16.2 \mathrm{~dB}$ at $\Delta_{1} / \gamma_{1}=7.0$ for $p_{\text {in }}=50 \mathrm{~mW}$.

Then we examine different effects of optical coupling strengths $J_{12}$ and $J_{23}$ by plotting in Figure 6 transmission coefficients $T_{31}$ and $T_{13}$ against detuning $\Delta_{1}$. Figures 6A,B show that a slight increase in $J_{12}$ will result in an evidently identical rising of $T_{31}$ and $T_{13}$ but leaving their peaks roughly unchanged in position. The main difference lies in that the upper branch of $T_{31}$ in Figure 6A exhibits a wider stable region than that in Figure $\mathbf{6 B}$, indicating that a larger $J_{12}$ helps to suppress quantum fluctuations arising from the non-linear optomechanical interaction. On the other hand, Figures 6C,D show that a slight increase in $J_{23}$ can also result in an evidently identical rising of $T_{31}$ and $T_{13}$, but their peaks become evidently closer to each other, leading to a narrowing of the

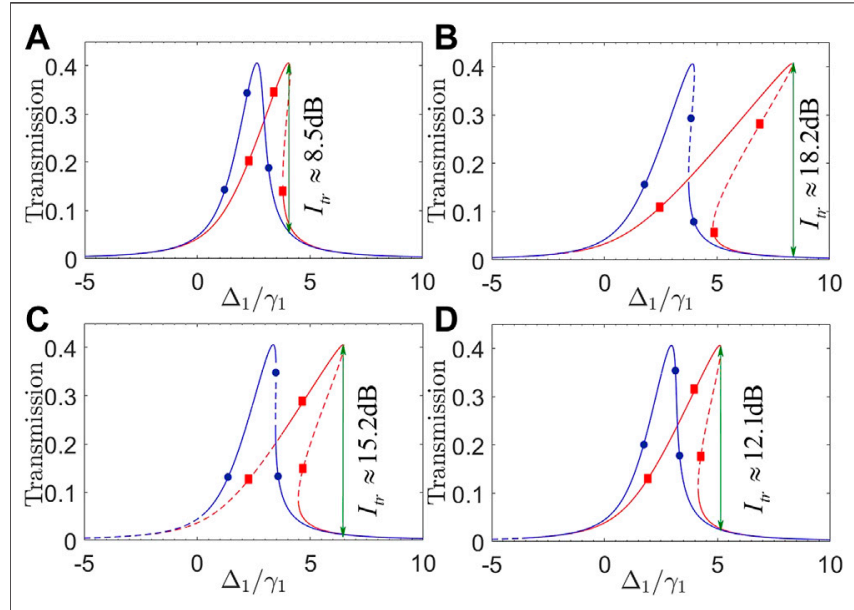

FIGURE 7 | (Color online) Transmission coefficients $T_{31}$ (red squares) and $T_{13}$ (blue circles) against detuning $\Delta_{1}$ with (A) $g / 2 \pi=0.9 \mathrm{MHz}$; (B) $g / 2 \pi=$ $1.6 \mathrm{MHz}$; (C) $\omega_{m} / 2 \pi=4.5 \mathrm{GHz}$; (D) $\omega_{m} / 2 \pi=6.5 \mathrm{GHz}$. Solid and dashed parts of each curve refer to stable and unstable regions, respectively. Other parameters are the same as in Figure 5, except $p_{i n}=20 \mathrm{~mW}$.

non-reciprocal bistable region as well as a reduction in the isolation ratio. These findings tell that a moderate $J_{23}$ and a larger $J_{12}$ are appropriate for attaining non-reciprocal bistable regions of wide enough stable upper branches and large enough isolation ratios.

Finally, we examine different effects of mechanical frequency $\omega_{m}$ and optomechanical coupling strength $g$ by plotting in Figure 7 transmission coefficients $T_{31}$ and $T_{13}$ against detuning $\Delta_{1}$. Comparing Figures $7 \mathbf{A}, \mathbf{B}$, we can see that both $T_{31}$ and $T_{13}$ remain unchanged in their peak values but clearly become more inclined toward $\Delta_{1}>0$ so as to yield wider bistable regions, with the increase in $g$. This then results in a wider non-reciprocal transmission region considering that $T_{31}$ is much more sensitive to $g$ and thus exhibits a much wider bistable region than $T_{13}$. Figures 7C,D show however that an increase in $\omega_{m}$ is helpful to reduce the unstable region of $T_{31}$ in its upper branch but meanwhile also results in a reduction of the bistable regions for both $T_{31}$ and $T_{13}$. These findings tell that one should choose a lower $\omega_{m}$ and a higher $g$ to enhance the non-linear optomechanical interaction required for attaining wider non-reciprocal transmission regions of high isolation ratios.

In figures, the bistable transmission non-reciprocity occurs as the two curves for $T_{31}$ and $T_{13}$ do not overlap in each plot. It is thus appropriate to roughly determine a non-reciprocal bandwidth as the absolute difference of two values of $p_{\text {in }}$ or $\Delta_{1}$ corresponding, respectively, to the peak of $T_{31}$ and that of $T_{13}$. This non-reciprocal bandwidth is a few or tens of $m W$ in Figures 2-4, while several times of $\gamma_{1}$ in Figures 5-7, depending on relevant parameters like $J_{12}, J_{23}, g$, and $\omega_{m}$. Note also that a dynamic reciprocity, referring to the fact that a (weak) backward noise, can also be transmitted with little loss in the presence of a (strong) forward signal of high transmission, typically exists in the non-reciprocal systems based on optical non-linearities [59]. 
Accordingly, one limitation of our optomechanical system may be that it cannot break the dynamic reciprocity because the transmission non-reciprocity arises from a bistable nonlinearity. This is clear by looking at Figures 2-4 where we have $T_{31} \neq T_{13}$ only when input power $p_{\text {in }}$ is not too small.

\section{CONCLUSION}

In summary, we have studied a four-mode optomechanical system for attaining the transmission non-reciprocity, in the presence of an optomechanically induced non-linearity, with respect to a driving field input from the left or right side. As the impedance-matching condition is broken, we find that transmission coefficients $T_{31}$ and $T_{13}$, plotted against input power $p_{\text {in }}$ or cavity detuning $\Delta_{1}$, may exhibit staggered bistable behaviors and therefore can work in the upper and lower branches, respectively. The isolation ratio of such a non-reciprocal transmission is viable to switch between $I_{t r}>0$ and $I_{t r}<0$ and can be improved in magnitude by modulating optical coupling strengths $J_{12,23}$ and detunings $\Delta_{1,3}$ to suppress the lower branches or enhance the upper branches. It is also viable to broaden the nonreciprocal bistable region in terms of $p_{\text {in }}\left(\Delta_{1}\right)$ by modulating optomechanical coupling strength $g$ and mechanical frequency $\omega_{m}$ in addition to $J_{12,23}$ and $\Delta_{1,3}\left(p_{i n}\right)$. But we should note that an increasing part of the upper branch may become unstable as the non-reciprocal region becomes wider, which restricts the tunable ranges of relevant parameters. Our results well extend the previous works on realizing non-reciprocal transmission in optomechanical systems and are instructive for designing non-reciprocal devices in optical networks based on coupled cavities.

\section{REFERENCES}

1. Teufel JD, Harlow JW, Regal CA, Lehnert KW. Dynamical Backaction of Microwave fields on a Nanomechanical Oscillator. Phys Rev Lett (2008) 101: 197203. doi:10.1103/PhysRevLett.101.197203

2. Aspelmeyer M, Kippenberg TJ, Marquardt F. Cavity Optomechanics. Rev Mod Phys (2014) 86:1391-452. doi:10.1103/RevModPhys.86.1391

3. Liu Y-C, Hu Y-W, Wong C-W, Xiao Y-F. Review of Cavity Optomechanical Cooling. Chin Phys B (2013) 22:114213. doi:10.1088/ 1674-1056/22/11/114213

4. He B. Quantum Optomechanics beyond Linearization. Phys Rev A (2012) 85: 063820. doi:10.1103/PhysRevA.85.063820

5. Cao C, Mi S-C, Gao Y-P, He L-Y, Yang D, Wang T-J, et al. Tunable HighOrder Sideband Spectra Generation Using a Photonic Molecule Optomechanical System. Sci Rep (2016) 6:1-8. doi:10.1038/srep22920

6. Xiong X-R, Gao Y-P, Liu X-F, Cao C, Wang T-J, Wang C. The Analysis of High-Order Sideband Signals in Optomechanical System. Sci China Phys Mech Astron (2018) 61:1-4. doi:10.1007/s11433-017-9187-2

7. Shi H-Q, Xie Z-Q, Xu X-W, Liu N-H. Unconventional Phonon Blockade in Multimode Optomechanical System. Acta Phys Sin (2018) 67:044203. doi:10.7498/aps.67.20171599

8. Marinković I, Wallucks A, Riedinger R, Hong S, Aspelmeyer M, Gröblacher S. Optomechanical bell Test. Phys Rev Lett (2018) 121:220404. doi:10.1103/ PhysRevLett.121.220404

9. Xu H, Lai D-G, Qian Y-B, Hou B-P, Miranowicz A, Nori F. Optomechanical Dynamics in the PT - and Broken- PT -symmetric regimesmathcalPT- and broken-mathcalPT-symmetric Regimes. Phys Rev A (2021) 104:053518. doi:10.1103/PhysRevA.104.053518

\section{DATA AVAILABILITY STATEMENT}

The original contributions presented in the study are included in the article/supplementary material; further inquiries can be directed to the corresponding authors.

\section{AUTHOR CONTRIBUTIONS}

The idea was first conceived by J-HW. BJ was responsible for the physical modeling, the numerical calculations, and writing most of the manuscript. J-HW contributed to writing the manuscript, JW verified results of the theoretical calculation, and DY contributed to the discussion of the results. DQ provided technical support in computer simulation.

\section{FUNDING}

This work is supported by the National Natural Science Foundation of China (12074061, 11674049, and 11874004), Science Foundation of Education Department of Jilin Province (JJKH20200557KJ), and Nature Science Foundation of Science and Technology Department of Jilin Province (20210101411JC).

\section{ACKNOWLEDGMENTS}

BJ thanks Luning Song, Yan Zhang, and Zhihai Wang for helpful discussions.

10. Vitali D, Gigan S, Ferreira A, Böhm HR, Tombesi P, Guerreiro A, et al. Optomechanical Entanglement between a Movable Mirror and a Cavity Field. Phys Rev Lett (2007) 98:030405. doi:10.1103/PhysRevLett.98.030405

11. Chen R-X, Shen L-T, Yang Z-B, Wu H-Z, Zheng S-B. Enhancement of Entanglement in Distant Mechanical Vibrations via Modulation in a Coupled Optomechanical System. Phys Rev A (2014) 89:023843. doi:10.1103/PhysRevA.89.023843

12. Liao J-Q, Wu Q-Q, Nori F. Entangling Two Macroscopic Mechanical Mirrors in a Two-Cavity Optomechanical System. Phys Rev A (2014) 89:014302. doi:10.1103/PhysRevA.89.014302

13. Yan X-B. Enhanced Output Entanglement with Reservoir Engineering. Phys Rev A (2017) 96:053831. doi:10.1103/PhysRevA.96.053831

14. He Q, Ficek Z. Einstein-podolsky-rosen Paradox and Quantum Steering in a Three-Mode Optomechanical System. Phys Rev A (2014) 89:022332. doi:10.1103/PhysRevA.89.022332

15. Zhang X-L, Bao Q-Q, Yang M-Z, Tian X-S. Entanglement Characteristics of Output Optical fields in Double-Cavity Optomechanics. Acta Phys Sin (2018) 67:104203. doi:10.7498/aps.67.20172467

16. Poot M, Herre SJ. Mechanical Systems in the Quantum Regime. Phys Rep (2012) 511:273-335. doi:10.1016/j.physrep.2011.12.004

17. He B, Yang L, Lin Q, Xiao M. Radiation Pressure Cooling as a Quantum Dynamical Process. Phys Rev Lett (2017) 118:233604. doi:10.1103/ PhysRevLett.118.233604

18. Chen H-J, Mi X-W. Normal Mode Splitting and Cooling in strong Coupling Optomechanical Cavity. Acta Phys Sin (2011) 60:124206-244. doi:10.7498/ aps.60.124206

19. Li Y, Wu L-A, Wang Z-D. Fast Ground-State Cooling of Mechanical Resonators with Time-dependent Optical Cavities. Phys Rev A (2011) 83: 043804. doi:10.1103/PhysRevA.83.043804 
20. Liu Z-Q, Hu C-S, Jiang Y-K, Su W-J, Wu H, Li Y, et al. Engineering Optomechanical Entanglement via Dual-Mode Cooling with a Single Reservoir. Phys Rev A (2021) 103:023525. doi:10.1103/PhysRevA.103.023525

21. Bai C, Hou B-P, Lai D-G, Wu D. Tunable Optomechanically Induced Transparency in Double Quadratically Coupled Optomechanical Cavities within a Common Reservoir. Phys Rev A (2016) 93:043804. doi:10.1103/PhysRevA.93.043804

22. Yan X-B. Optomechanically Induced Transparency and Gain. Phys Rev A (2020) 101:043820. doi:10.1103/PhysRevA.101.043820

23. Lü H, Wang C, Yang L, Jing H. Optomechanically Induced Transparency at Exceptional Points. Phys Rev Appl (2018) 10:014006. doi:10.1103/ PhysRevApplied.10.014006

24. Hou B-P, Wei L-F, Wang S-J. Optomechanically Induced Transparency and Absorption in Hybridized Optomechanical Systems. Phys Rev A (2015) 92: 033829. doi:10.1103/PhysRevA.92.033829

25. Qu K, Agarwal GS. Phonon-mediated Electromagnetically Induced Absorption in Hybrid Opto-Electromechanical Systems. Phys Rev A (2013) 87:031802. doi:10.1103/PhysRevA.87.031802

26. Zhang J-Q, Li Y, Feng M, Xu Y. Precision Measurement of Electrical Charge with Optomechanically Induced Transparency. Phys Rev A (2012) 86:053806. doi:10.1103/PhysRevA.86.053806

27. Brunner N, Cavalcanti D, Pironio S, Scarani V, Wehner S. Bell Nonlocality. Rev Mod Phys (2014) 86:419-78. doi:10.1103/RevModPhys.86.419

28. Lü X-Y, Jing H, Ma J-Y, Wu Y. PT-Symmetry-Breaking Chaos in Optomechanics. Phys Rev Lett (2015) 114:253601. doi:10.1103/ PhysRevLett.114.253601

29. Margueritat J, Virgone-Carlotta A, Monnier S, Delanoë-Ayari H, Mertani HC, Berthelot A, et al. High-frequency Mechanical Properties of Tumors Measured by Brillouin Light Scattering. Phys Rev Lett (2019) 122:018101. doi:10.1103/ PhysRevLett.122.018101

30. Song L-N, Zheng Q, Xu X-W, Jiang C, Li Y. Optimal Unidirectional Amplification Induced by Optical Gain in Optomechanical Systems. Phys Rev A (2019) 100:043835. doi:10.1103/PhysRevA.100.043835

31. Xu X-W, Song L-N, Zheng Q, Wang Z-H, Li Y. Optomechanically Induced Nonreciprocity in a Three-Mode Optomechanical System. Acta Phys Sin (2018) 98:063845. doi:10.1103/PhysRevA.98.063845

32. Zhang L-W, Li X-L, Yang L. Optical Nonreciprocity with Blue-Detuned Driving in Two-Cavity Optomechanics. Acta Phys Sin (2019) 68:170701-9. doi:10.7498/aps.68.20190205

33. Miri M-A, Ruesink F, Verhagen E, Alù A. Optical Nonreciprocity Based on Optomechanical Coupling. Phys Rev Appl (2017) 7:064014. doi:10.1103/ PhysRevApplied.7.064014

34. Li E-Z, Ding D-S, Yu Y-C, Dong M-X, Zeng L, Zhang W-H, et al. Experimental Demonstration of Cavity-free Optical Isolators and Optical Circulators. Phys Rev Res (2020) 2:033517. doi:10.1103/PhysRevResearch.2.033517

35. Li B, Özdemir ŞK, Xu X-W, Zhang L, Kuang L-M, Jing H. Nonreciprocal Optical Solitons in a Spinning Kerr Resonator. Phys Rev A (2021) 103:053522. doi:10.1103/PhysRevA.103.053522

36. Verhagen E, Alù A. Optomechanical Nonreciprocity. Nat Phys (2017) 13: 922-4. doi:10.1038/nphys4283

37. Lira H, Yu Z, Fan S, Lipson M. Electrically Driven Nonreciprocity Induced by Interband Photonic Transition on a Silicon Chip. Phys Rev Lett (2012) 109: 033901. doi:10.1103/PhysRevLett.109.033901

38. Manipatruni S, Robinson JT, Lipson M. Optical Nonreciprocity in Optomechanical Structures. Phys Rev Lett (2009) 102:213903. doi:10.1103/PhysRevLett.102.213903

39. Gridnev VN. Nonreciprocal Reflection of Light from Antiferromagnets. Jetp Lett (1996) 64:110-3. doi:10.1134/1.567141

40. Lu X, Cao W, Yi W, Shen H, Xiao Y. Nonreciprocity and Quantum Correlations of Light Transport in Hot Atoms via Reservoir Engineering. Phys Rev Lett (2021) 126:223603. doi:10.1103/PhysRevLett.126.223603

41. Wang Y-P, Rao J-W, Yang Y, Xu P-C, Gui Y-S, Yao B-M, et al. Nonreciprocity and Unidirectional Invisibility in Cavity Magnonics. Phys Rev Lett (2019) 123: 127202. doi:10.1103/PhysRevLett.123.127202

42. Lai D-G, Huang J-F, Yin X-L, Hou B-P, Li W, Vitali D, et al. Nonreciprocal Ground-State Cooling of Multiple Mechanical Resonators. Phys Rev A (2020) 102:011502. doi:10.1103/PhysRevA.102.011502

43. Khanikaev AB, Mousavi SH, Shvets G, Kivshar YS. One-way Extraordinary Optical Transmission and Nonreciprocal Spoof Plasmons. Phys Rev Lett (2010) 105:126804. doi:10.1103/PhysRevLett.105.126804
44. Fang K, Luo J, Metelmann A, Matheny MH, Marquardt F, Clerk AA, et al. Generalized Non-reciprocity in an Optomechanical Circuit via Synthetic Magnetism and Reservoir Engineering. Nat Phys (2017) 13:465-71. doi:10.1038/nphys4009

45. Dzyaloshinskii I, Papamichail EV. Nonreciprocal Optical Rotation in Antiferromagnets. Phys Rev Lett (1995) 75:3004-7. doi:10.1103/ PhysRevLett.75.3004

46. Ni X, He C, Sun X-C, Liu X-P, Lu M-H, Feng L, et al. Topologically Protected One-Way Edge Mode in Networks of Acoustic Resonators with Circulating Air Flow. New J Phys (2015) 17:053016. doi:10.1088/13672630/17/5/053016

47. Yu H, Wang Z, Wu J-H. Entanglement Preparation and Nonreciprocal Excitation Evolution in Giant Atoms by Controllable Dissipation and Coupling. Phys Rev A (2021) 104:013720. doi:10.1103/ PhysRevA.104.013720

48. Du L, Cai M-R, Wu J-H, Wang Z, Li Y. Single-photon Nonreciprocal Excitation Transfer with Non-markovian Retarded Effects. Phys Rev A (2021) 103:053701. doi:10.1103/PhysRevA.103.053701

49. Zhang S, Hu Y, Lin G, Niu Y, Xia K, Gong J, et al. Thermal-motion-induced Non-reciprocal Quantum Optical System. Nat Photon (2018) 12:744-8. doi:10.1038/s41566-018-0269-2

50. Xia K, Nori F, Min X. Cavity-free Optical Isolators and Circulators Using a Chiral Cross-Kerr Nonlinearity. Phys Rev Lett (2018) 121:203602. doi:10.1103/ PhysRevLett.121.203602

51. Hu Y, Zhang S, Qi Y, Lin G, Niu Y, Gong S. Multiwavelength Magnetic-free Optical Isolator by Optical Pumping in Warm Atoms. Phys Rev Appl (2019) 12: 054004. doi:10.1103/PhysRevApplied.12.054004

52. Paraïso TK, Kalaee M, Zang L, Pfeifer H, Marquardt F, Painter O. Positionsquared Coupling in a Tunable Photonic crystal Optomechanical Cavity. Phys Rev X (2015) 5:041024. doi:10.1103/PhysRevX.5.041024

53. DeJesus EX, Kaufman C. Routh-hurwitz Criterion in the Examination of Eigenvalues of a System of Nonlinear Ordinary Differential Equations. Phys Rev A (1987) 35:5288-90. doi:10.1103/PhysRevA.35.5288

54. Paternostro M, Gigan S, Kim MS, Blaser F, Böhm HR, Aspelmeyer M. Reconstructing the Dynamics of a Movable Mirror in a Detuned Optical Cavity. New J Phys (2006) 8:107. doi:10.1088/1367-2630/8/ $6 / 107$

55. Gardiner CW, Collett MJ. Input and Output in Damped Quantum Systems: Quantum Stochastic Differential Equations and the Master Equation. Phys Rev A (1985) 31:3761-74. doi:10.1103/PhysRevA.31.3761

56. Agarwal GS, Huang S. Optomechanical Systems as Single-Photon Routers. Phys Rev A (2012) 85:021801. doi:10.1103/PhysRevA.85.021801

57. Aldana S, Bruder C, Nunnenkamp A. Equivalence between an Optomechanical System and a Kerr Medium. Phys Rev A (2013) 88:043826. doi:10.1103/PhysRevA.88.043826

58. Fabre C, Pinard M, Bourzeix S, Heidmann A, Giacobino E, Reynaud S. Quantum-noise Reduction Using a Cavity with a Movable Mirror. Phys Rev A (1994) 49:1337-43. doi:10.1103/PhysRevA.49.1337

59. Shi Y, Yu Z, Fan S. Limitations of Nonlinear Optical Isolators Due to Dynamic Reciprocity. Nat Photon (2015) 9:388-92. doi:10.1038/nphoton.2015.79

Conflict of Interest: The authors declare that the research was conducted in the absence of any commercial or financial relationships that could be construed as a potential conflict of interest.

Publisher's Note: All claims expressed in this article are solely those of the authors and do not necessarily represent those of their affiliated organizations, or those of the publisher, the editors, and the reviewers. Any product that may be evaluated in this article, or claim that may be made by its manufacturer, is not guaranteed or endorsed by the publisher.

Copyright $\odot 2022$ Jiang, Yan, Wang, $Q u$ and $W u$. This is an open-access article distributed under the terms of the Creative Commons Attribution License (CC BY). The use, distribution or reproduction in other forums is permitted, provided the original author(s) and the copyright owner(s) are credited and that the original publication in this journal is cited, in accordance with accepted academic practice. No use, distribution or reproduction is permitted which does not comply with these terms. 